\title{
Video Transmission over an Enhancement Approach Of IEEE802.11e
}

\author{
Abdirisaq M. Jama and Othman O. khalifa \\ Faculty of Engineering \\ International Islamic University \\ Malaysia
}

\author{
Diaa Eldein Mustafa Ahmed \\ Faculty of Computer Science and Information \\ Technology, Sudan University for Science and \\ Technology, Sudan
}

\begin{abstract}
Multimedia Video transmission is over Wireless Local Area Networks is expected to be an important component of many emerging multimedia applications. However, Wireless networks will always be bandwidth limited compared to fixed networks due to background noise, limited frequency spectrum, and varying degrees of network coverage and signal strength One of the critical issues for multimedia applications is to ensure that the Quality of Service (QoS) requirement to be maintained at an acceptable level. Modern mobile devices are equipped with multiple network interfaces, including 3G/LTE WiFi. Bandwidth aggregation over LTE and WiFi links offers an attractive opportunity of supporting bandwidth-intensive services, such as high-quality video streaming, on mobile devices. Achieving effective bandwidth aggregation in wireless environments raises several challenges related to deployment, link heterogeneity, Network congestion, network fluctuation, and energy consumption. In this work, an overview of schemes for video transmission over wireless networks is presented where an acceptable quality of service (QoS) for video applications required realtime video transmission is achieved.
\end{abstract}

Keywords: Video coding, video compression, wireless video transmission, Wireless Networks

\section{INTRODUCTION}

Video Transmission has been an important media for communications and entertainment for many decades. Initially video was captured and transmitted in analog form. The advent of digital integrated circuits and computers led to the digitization of video, and digital video enabled a revolution in the compression and communication of video. Video compression became an important area of research in the late 1980's and 1990's and enabled a variety of applications including video storage on DVD's and Video-CD's, video broadcast over digital cable, satellite and terrestrial (over-theair) digital television (DTV), and video conferencing and videophone over circuit-switched networks. The growth and popularity of the Internet in the mid-1990's motivated video communication over best-effort packet networks [1][2][3]. It is complicated by a number of factors including unknown and time -varying bandwidth, delay, and losses, as well as many additional issues such as how to fairly share the network resources amongst many flows and how to efficiently perform one-to-many communication for popular content. Figure 1 shows Internet Video Streaming Architecture where Raw video and audio data are pre-compressed by video compression and audio compression algorithms and then saved in storage devices[4][5].

Upon the client's request, a streaming server retrieves compressed video/audio data from storage devices and then the application-layer QoS control module adapts the video/audio bit-streams according to the network status and QoS requirements. After the adaptation, the transport protocols packetize the compressed bitstreams and send the video/audio packets to the Internet. Packets may be dropped or experience excessive delay inside the Internet due to congestion. For packets that are successfully delivered to the receiver, they first pass through the transport layers and then are processed by the application layer before being decoded at the video/audio decoder. With respect to the real-time transmission of video streams, the transmitting delay should be minimal. The high transmitting delay may cause the video packets not to been decoded. Adjustment of the bit rates of video stream is required for a reliable video transmission [6][7][8] . To achieve synchronization between video and audio presentations, media synchronization mechanisms are required.

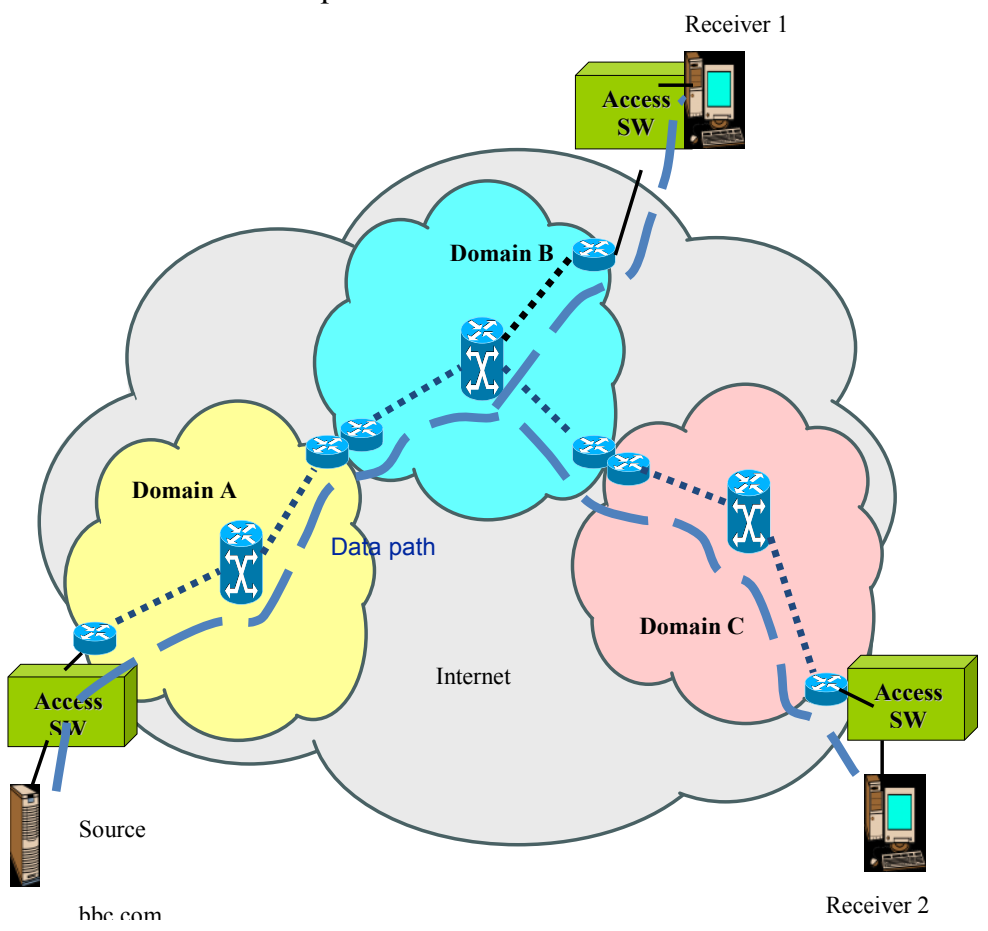

Figure .1 Internet Video Streaming

Upon the client's request, a streaming server retrieves compressed video/audio data from storage devices and then the application-layer QoS control module adapts the video/audio bit-streams according to the network status and QoS requirements [9][10][11]. After the 
adaptation, the transport protocols packetize the compressed bit-streams and send the video/audio packets to the Internet [12][13][14]. Packets may be dropped or experience excessive delay inside the Internet due to congestion. For packets that are successfully delivered to the receiver, they first pass through the transport layers and then are processed by the application layer before being decoded at the video/audio decoder. With respect to the real-time transmission of video streams, the transmitting delay should be minimal. The high transmitting delay may cause the video packets not to been decoded. Adjustment of the bit rates of video stream is required for a reliable video transmission. To achieve synchronization between video and audio presentations, media synchronization mechanisms are required.

\section{Taxonomy of Video Applications}

There exist very diverse ranges of video communication. Video communication applications may be unicast, multicast, broadcast or anycast. The video may be pre-encoded (stored) or real- time encoded (e.g. videoconferencing applications). The communication channel may be static or dynamic, reserved or not, packet switched or circuit switched, may support some quality of service or may only provide best effort service. The specific properties of a video communication application strongly influence the design of the system. We continue by briefly discussing these properties.

\subsubsection{Point-to-point, multicast, broadcast and anycast communications:}

Probably the most popular form of video communication is one-to-many (basically one-to-all) communication or broadcast communication, where the most well-known example is broadcast television. Broadcast wastes bandwidth by sending the data to the whole network. It can also needlessly slow the performance of client machines because each client must process the broadcasted data whether or not the service is of interest. The main challenge for broadcasting is the scalability problem. Receivers may experience different channel characteristics, and the sender must cope with all the receivers. Another common form of communication is pointto-point or one-to-one communication, e.g. videophone and unicast video streaming over the Internet. In point -to-point communications, an important property is whether or not there is a back channel between the receiver and sender. If a back channel exists, the receiver can provide feedback to the sender which the sender can then use to adapt its processing. Unicast wastes bandwidth by sending multiple copies of the data. Another form of communication with properties that lie between point- to- point and broadcast is multicast. Multicast is a one-to-many communication, but it is not one-to-all as in broadcast. An example of multicast is IP- Multicast over the Internet. To communicate to multiple receivers, multicast is more efficient than multiple unicast connections (i.e. one dedicated unicast connection to each client), and overall multicast provides many of the same advantages and disadvantages as broadcast. The anycasting communication paradigm is designed to support server replications to easily select and communicate with the best server, according to some performance or policy criteria, in a group of contentequivalent servers.

\subsubsection{Real-time encoding versus pre-encoded (stored)} video

Video may be captured and encoded for real-time communication, or it may be pre-encoded and stored for later viewing. Interactive applications are one example of applications which require real-time encoding, e.g. videophone, video conferencing, or interactive games. In many applications video content is pre-encoded and stored for later viewing. The video may be stored locally or remotely. Examples of local storage include DVD and Video CD, and examples of remote storage include video-on-demand (VOD), and video streaming over the Internet (e.g. as provided by Real Networks and Microsoft). Pre-encoded video

has the advantage that it does not require a real-time encoding constraint, which enables more efficient encoding. On the other hand, it provides limited flexibility as, for example, the pre-encoded video can not be significantly adapted clients that support different display capabilities than that used in the original encoding.

\subsubsection{Interactive versus Non-interactive Applications}

Interactive applications have real-time data delivery constraints. The data sent has time bounded usefulness, after this time the received data is useless.

Various applications can be mapped onto axes of packet loss and one-way delay. The size and shape of the boxes provide a general indication of the limit of delay and information loss tolerable for each application class. The following classes of applications can be recognized:

- Interactive video applications. They need a few milliseconds of transfer delay such as conversational voice and video, interactive games, etc.

- Responsive video applications. Typically, these applications response in few seconds, so that human does not need to wait for a long time, such as voice and video messaging, transactions, Web, etc.

- Timely video application. The transfer delay can be about some second, such as streaming audio and video.

- Non-critical video application. The transfer delay is not a critical for those applications, such as audio and video download service. From loss point of view, we can find two types of applications:

- Error sensitive video applications such as highly compressed video.

- Error insensitive video applications such as noncompressed video.

The loss has a direct impact on the quality of the information finally presented to the user, whether it is voice, image, video or data. In this context, loss is not limited to the effects of bit errors or packet loss during transmission, but also includes the effects of any degradation introduced by media coding.

\section{VIDEO TRANSMISIIN CHALLENGES}

There are many different types of video transmission applications such as Video on Demand (VoD), real-time and near real-time video streaming and MMS. In addition video streams can be streamed with a one-to-one (i.e. Unicast) or one-to-many (i.e. Multicast/Broadcast). There is also a huge range of video content possible. For example, ask yourself what is a typical video clip? It is difficult even to characterize the characteristics of the content in terms of how much action and detail is contained in a video clip. Before video can be transmitted over the network, it must first be encoded. There are a huge number of ways in which video can be encoded these include the choice of codec (i.e. MPEG-2, MPEG-4, 
H.264, AVI, WMV etc.), the target bit rate, the frame rate, equalization parameter, the resolution and so on. The choice of these parameters will affect the delivery of the video on the network. Once the video has been encoded, it is then transmitted/streamed using a streaming server. The server can transmit the video in a number of ways using various transmission protocols and packetization schemes. The client periodically sends feedback to the server telling the server how much information has been received. The server uses this feedback to adapt the transmitted video stream so as to minimize the any negative effects of congestion in the network might have on the video stream. The ability of the server to optimally adapt the video stream depends on the frequency of the feedback and the relevance, usefulness, and accuracy of the feedback information [15][16]. There are a number of different techniques that can be used in the server to adapt the video quality including rate control, rate shaping, frame dropping, and stream switching. Finally, to add to the difficulties of video streaming, there are no accepted metrics to calculate video quality so as to correlate to the Human Visual System (HVS) or in other words human perception, e.g. PSNR, VQM, MPQM, PVQ etc [6]. There is a strong demand in modern societies for pioneering ICT services that will support modern social infrastructures. Emerging new techniques in the fields of wireless communication, network coding and video transmission, which can be used as a base for creating smart services that would serve people's everyday life in modern societies? Typical example of such services is video surveillance over wireless networks to support traffic monitoring, fire detection and real-time events (such as natural disasters) broadcasting for the societies of the smart cities, real time monitoring of patients in ICU.

\section{VIDEO QUALITY EVALUATION}

Several factors, such as network delay, packet loss etc., may lead to loss of video data that can distort the video sequence. Two types of methodologies have become popular that can measure the distortion: objective assessment and subjective assessment. We describe these approaches in the following text.

\subsection{Objective Assessment}

Objective Assessment methods use algorithms to measure the distortion in a given video sequence. These algorithms are fast and very easy to use [17]. Most of these algorithms require the original signal in order to compare it with the distorted signal. One of the most popular methods is to use the Peak Signal-to-Noise Ratio (PSNR) measure.

PSNR gives the distortion between the original and the processed (impaired) versions of a video sequence. Let's say that we have two sequences: $\mathrm{S}$ (original) and $\mathrm{S}^{\prime}$ (impaired). $\mathrm{S}(\mathrm{x}, \mathrm{y}, \mathrm{k})$ is the luminance of a pixel at position $\mathrm{x}, \mathrm{y}$ in frame $\mathrm{k}$ from the original sequence and $\mathrm{S}^{\prime}(\mathrm{x}, \mathrm{y}, \mathrm{k})$ is the luminance of a pixel at the corresponding position in the impaired version. The sequences are $\mathrm{K}$ frames long, the frame size is $\mathrm{M} * \mathrm{~N}$ pixels, and each pixel luminance is represented with 8 bits. The Mean Square Error is first obtained with the Equation.1.

$$
M S E=\frac{1}{K M N} \sum_{k=1}^{K} \sum_{y=1}^{M} \sum_{x=1}^{N}\left[S(x, y, k)-S^{\prime}(x, y, k)\right]^{2}
$$

The MSE is the cumulative squared error between the original and the impaired images. A lower MSE means a smaller error. The PSNR is then computed with the following equation $2 .:-$

$$
P S N R=20 \log _{10} \frac{255}{\sqrt{M S E}}
$$

The unit of the PSNR is a decibel value (dB), 255 is the maximum possible pixel value of the image. When the pixels are represented using 8 bits per sample. Typical values for the PSNR image and video compression are between 30 and $50 \mathrm{~dB}$, where higher is better. Acceptable values for PSNR are considered to be about $28 \mathrm{~dB}$ to $35 \mathrm{~dB}$.

\subsection{Subjective Assessment}

Subjective assessment methods are supposed to be the best indicators of the video quality, for a video that will be watched by humans, because the assessment is done by real humans. In general a distorted sequence, in addition to the original sequence, is shown to the human subjects and they are asked to give a score to the sequence. Later, the scores from several subjects are statistically processed to give a mean score (the MOS or Mean Opinion Score) for that particular distorted sequence.

The ITU-R Recommendation (ITU-R, 2002) defines several standard methods and procedures for the subjective quality assessment of television pictures. One of the methods is called Double-Stimulus Impairment Scale (DSIS). In DSIS method, an assessor is first presented with the original video sequence, and then he is shown the distorted version. The assessor rates the degree of the impairment of the second image having the reference in mind. This is repeated with several pairs of sequences. The score for each sequence is taken from the impairment scale shown in Table 1.

Table 1 Impairment scale

\begin{tabular}{|c|c|c|}
\hline $\begin{array}{c}\text { Number } \\
\text { Score }\end{array}$ & Impairment Scale & Quality of Scale \\
\hline 5 & Imperceptible & Excellent \\
\hline 4 & $\begin{array}{c}\text { Perceptible, but not } \\
\text { Annoying }\end{array}$ & Good \\
\hline 3 & Slightly Annoying & Fair \\
\hline 2 & Annoying & Poor \\
\hline 1 & Very Annoying & Unsatisfactory \\
\hline
\end{tabular}

\section{CONTENT DELIVERY CHOICE IMPLICATIONS}

Each delivery technique has some inherent advantages and disadvantages. The selection of a means of delivery by training and education organizations should be primarily based on providing the best viewing experience to the learner as possible for a given instructional design. Familiarity with the various strengths and weaknesses of HTTP streaming, RTSP streaming, and CD content distribution methods are essential [18][19].

\subsection{Streaming Quality}

Between HTTP and RTSP streaming techniques, HTTP streaming usually permits content providers the ability to provide higher data rates. These higher data delivery rates permit higher quality files to be made available to viewers. The disadvantage of having the ability to support higher data delivery rates is the lengthy download times associated with the files. Additionally, viewers must be willing to wait for these files as well, often times needing a high-speed connection to endure the longer download times [20][21][22].

The HTTP streaming method guarantees the delivery of all of a given video files data, no matter how long it takes. The implication is there will be no dropped frames or 
missing information data that will lead to picture quality degradation. With RSTP streaming, there is no guarantee for the complete delivery of data. Consequently, viewers may experience dropped frames, excessive pixilation of images, or "jerky" motions if the network cannot deliver all of the data on time. If the network becomes overly congested, viewers may be unable to view or hear all of the data intended for them. However, with RSTP streaming, viewers will experience what they do see at the intended time; similar to a broadcast. Depending on the type of training and education being offered, missing some of the data, some of the time, may become unacceptable from a learning perspective.

For best picture quality, the CD or DVD will provide the largest and richest quality pictures. Most of the streaming methods are designed to deliver a smaller picture, approximately $240 \times 180$, at 12 to 15 frames per second. Because there is no network transfer involved with a $\mathrm{CD}$ or DVD, picture quality can be as large as $720 \times 480$, at 30 frames per second. If picture quality of video multimedia is of paramount importance in the instructional design of a given the training and education module, then CDs and DVDs are the delivery means of choice.

\subsection{File Size and Performance}

For individual video files longer than five minutes, RTSP streaming is usually a better choice than HTTP streaming. When downloading larger files, HTTP streaming can present problems for viewer connecting to the network without a high speed connection. Additionally, those viewers lacking adequate hard drive storage space and system processor speeds on their local machines tend to be frustrated with HTTP streaming architectures. Simply, the files take too long to download and users become impatient waiting the video to play. With RTSP streaming, there is only a small "priming" file to download before the entire video file begins to play. Under an RTSP streaming architecture, viewers can easily fast forward ahead through a video file and only have to wait a few seconds until the video playback begins to play at the new start point. Such functionality is not possible with HTTP streaming. With HTTP streaming, viewers cannot randomly access portions of a particular clip without downloading the entire file first.

Both types of streaming are suitable depending on the instructional design of a given course. If the course is supported by videos that are most likely to be watched once, RTSP streaming is suitable. However, if it is anticipated that students will watch the video repeatedly, viewing the file on a CD or DVD will provide a more satisfying experience.

\section{IEEE802.11E PERFORMANCE ANALYSIS FOR VIDEO TRANSMISSION}

In this work, Networks were designed for non-real time traffic, like data are today being used to support real-time applications like Video streaming which are inherently different from data traffic. Video applications have very different requirements and characteristics compared to data traffic. Packet-loss affects the quality of video and degrades the user experience. End-toend delay is also an important requirement, similar to that the throughput and bandwidth requirement is important. The traffic characteristics of the non-video flows were used in the simulations. The non-video flows were chosen to provide a mix of traffic types to compete in the medium \& increase the network load since all the stations share the access to the same channel to evaluate the performance analysis.

In this Scenario, video is given higher priority that the other video traffics; Hence, video gets faster access to the medium.

Figure 2 shows the network topology used for the simulation experiments of the first and second scenario which is a single AP with thee traffic flows.

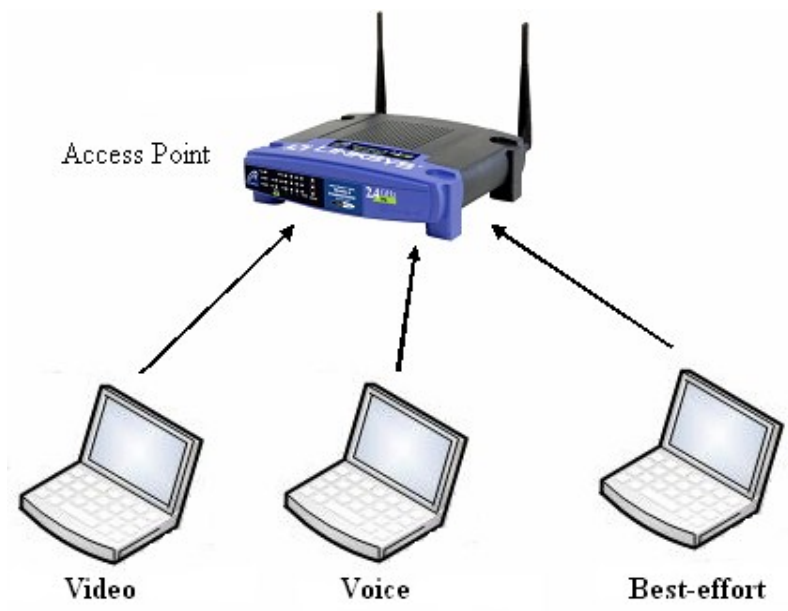

Figure 2 Three traffic flows

This scenario demonstrates three data flows. The number of mobile stations was increased from 3 to 15 to increase the network load. Three stations are added every simulation and each one of them transmits different data flow than others such as video, voice or best-effort data flow. This scenario is to calculate the throughput, delay and packet loss characteristics with the variation of number of stations.

For the second scenario is similar to the first one except that the number of mobile stations was increased from 3 to 9 , there are 3 groups of stations with 3 stations each. The first group transmits video flow, while the second transmits voice flow, and the third transmits best-effort data flow. Relatively, delay and packet loss are calculated under the variation of the topology where the stations are moving from 100 to 1000 square meters. This is considered as a very difficult scenario and it may be used to design hotspots under different conditions. Table 2 shows the IEEE802.11e MAC parameters values used in the simulation for the two scenarios.

Table 2 IEEE802.11e MAC Parameters

\begin{tabular}{|l|l|}
\hline Parameter & Value \\
\hline Slot time & $20 \mathrm{us}$ \\
\hline Beacon interval & $100 \mathrm{~ms}$ \\
\hline Fragmentation threshold & 1024 Bytes \\
\hline RTS threshold & 500 Bytes \\
\hline SIFS & $10 \mathrm{us}$ \\
\hline PIFS & $40 \mathrm{us}$ \\
\hline DIFS & $50 \mathrm{us}$ \\
\hline MSDU (Voice and Video) & $60 \mathrm{~ms}$ \\
\hline MSDU (data) & $200 \mathrm{~ms}$ \\
\hline Retry limit & 7 \\
\hline TXOP limit & $3428 \mathrm{us}$ \\
\hline CAP rate & $21 \mathrm{us}$ \\
\hline CAP max & $8000 \mathrm{us}$ \\
\hline CAP timer & $5120 \mathrm{us}$ \\
\hline
\end{tabular}


The number of mobile stations is increased from 3 to 15 with 3 stations at a time to increase the network load. As mentioned in the introduction of this simulation, every three QoS stations transmit three different types of flows (video, voice and best-effort data) to the same destination, which is the access point, and the PHY data rate is set $11 \mathrm{Mbps}$. Table 3 shows the simulation parameters used in the first \& second scenario.

Table 3 Enhanced EDCA Simulation Parameters

\begin{tabular}{|l|l|l|l|}
\hline $\begin{array}{l}\text { Simulation } \\
\text { Parameter }\end{array}$ & Video & Voice & $\begin{array}{l}\text { Best } \\
\text { effort }\end{array}$ \\
\hline Transport Protocol & UDP & UDP & UDP \\
\hline $\mathrm{CW}_{\min }$ & 3 & 7 & 15 \\
\hline $\mathrm{CW}_{\max }$ & 7 & 15 & 1023 \\
\hline AIFSN & 1 & 2 & 3 \\
\hline Packet Size (bytes) & 1028 & 160 & 1500 \\
\hline Packet Interval (ms) & 10 & 20 & 12.5 \\
\hline Data rate (kbps) & 822.40 & 64 & 960 \\
\hline
\end{tabular}

All the simulation results are averaged over five simulations, with random starting time for each flow. There is a variation in the channel load by increasing the number of active QoS stations from 3 to 15 with 3 stations at a time. All stations are in the range of each other.

Table 4 shows the original IEEE802.11e parameters used in the first scenario \& second scenario

Table 4 Original IEEE802.11e EDCA Simulation Parameters

\begin{tabular}{|l|l|l|l|}
\hline $\begin{array}{l}\text { Simulation } \\
\text { Parameter }\end{array}$ & Video & Voice & $\begin{array}{l}\text { Best-effort } \\
\text { Data }\end{array}$ \\
\hline $\mathrm{CW}_{\min }$ & 7 & 15 & 31 \\
\hline $\mathrm{CW}_{\max }$ & 15 & 31 & 1023 \\
\hline $\mathrm{AIFSN}$ & 2 & 3 & 4 \\
\hline
\end{tabular}

Results are based on the three basic performance metrics (Throughput, delay and packet loss) for the different access categories (video, voice and best-effort data). These metrics were selected due to their great effect on the IEEE802.11e performance for QoS support.

\section{DEMONSTRATION RESULTS}

In this section, a few simulation results of the two scenarios respectively as a comparative performance analysis of IEEE802.11e WLAN protocol are presented. These results include throughput, average end-to-end delay and packet loss. It also provides a detailed explanation of the behaviour of IEEE802.11e supported by graphs. The number of mobile stations is increased from 3 to 15 with 3 stations at a time to increase the network load. As mentioned in the introduction of this simulation, every three QoS stations transmit three different types of flows (video, voice and best-effort data) to the same destination, which is the access point, and the PHY data rate is set 11 Mbps. Table 4 shows the simulation parameters used in this scenario.

Table 4 Enhanced EDCA Simulation Parameters

\begin{tabular}{|c|c|c|c|}
\hline $\begin{array}{c}\text { Simulation } \\
\text { Parameter }\end{array}$ & Video & Voice & Best effort \\
\hline Transport Protocol & UDP & UDP & UDP \\
\hline $\mathrm{CW}_{\min }$ & 3 & 7 & 15 \\
\hline $\mathrm{CW}_{\max }$ & 7 & 15 & 1023 \\
\hline AIFSN & 1 & 2 & 3 \\
\hline $\begin{array}{c}\text { Packet Size } \\
\text { (bytes) }\end{array}$ & 1028 & 160 & 1500 \\
\hline $\begin{array}{c}\text { Packet Interval } \\
\text { (ms) }\end{array}$ & 10 & 20 & 12.5 \\
\hline \begin{tabular}{c} 
Data rate (kbps) \\
\hline
\end{tabular} & 822.40 & 64 & 960 \\
\hline
\end{tabular}

All the simulation results are averaged over five simulations, with random starting time for each flow. There is a variation in the channel load by increasing the number of active QoS stations from 3 to 15 with 3 stations at a time. All stations are in the range of each other. Table 5 shows the original IEEE802.11e parameters used in this scenario.

Table 5 Original IEEE802.11e EDCA Simulation Parameters for the first \& second scenario

\begin{tabular}{|c|c|c|c|}
\hline $\begin{array}{c}\text { Simulation } \\
\text { Parameter }\end{array}$ & Video & Voice & $\begin{array}{c}\text { Best-effort } \\
\text { Data }\end{array}$ \\
\hline $\mathrm{CW}_{\min }$ & 7 & 15 & 31 \\
\hline $\mathrm{CW}_{\max }$ & 15 & 31 & 1023 \\
\hline $\mathrm{AIFSN}$ & 2 & 3 & 4 \\
\hline
\end{tabular}

Results are based on the three basic performance metrics (Throughput, delay and packet loss) for the different access categories (video, voice and best-effort data). These metrics were selected due to their great effect on the IEEE802.11e performance for QoS support. The following analysis focuses the throughput results for the first scenario, which is shown in Figure 3.

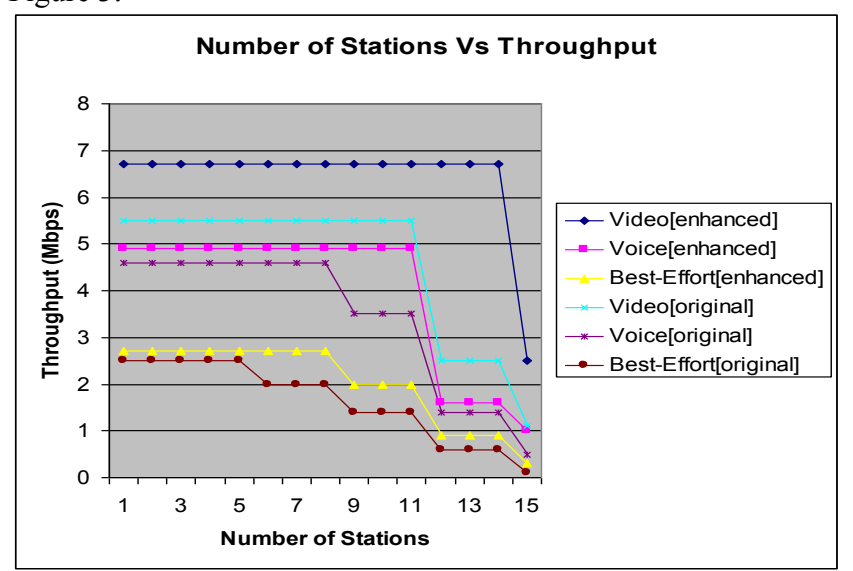

Figure 3 Effect of network load on throughput for different access categories (video, voice and best effort data) using original \& enhanced EDCA values 
The graph illustrates the effect of increasing the number of active QoS stations transmitting data to the access point on the throughput values for the three data flows. The sending rate in this simulation is $11 \mathrm{Mbps}$, while the $\mathrm{CW}_{\min }$ and $\mathrm{CW}_{\max }$ size and AIFSN values as stated in Table $4 \& 5$. In comparison, Figure 3 illustrates the effect of increasing the number of active QoS stations transmitting data to the access point on the throughput values for the three data flows using IEEE802.11e standard (IEEE, 2003) CW size and AIFSN values shown in Table 5 .

Enhanced CW size and AIFSN values provide better results considering the video and voice flows, this is clearly observed from Figure 3. In both cases, it is clearly seen from the graphs that IEEE802.11e provides service differentiation for different priorities when the system is heavily loaded by increasing the number of stations. When the number of stations is 3 or 6 , all the data flows have equal channel capacity. However, in the case of 9,12 , and 15 stations, the channel is reserved for higher priority data flows. As explained in the beginning of this chapter, video flow has the highest priority among the others, while the best effort data flow has the lowest priority.

The average end-to-end delay is another important performance metric that should be taken into account. Figure 4 represent the results obtained from the simulations using the enhanced CW size and AIFSN values.

\section{Number of Nodes Vs Average end-to-end delay}

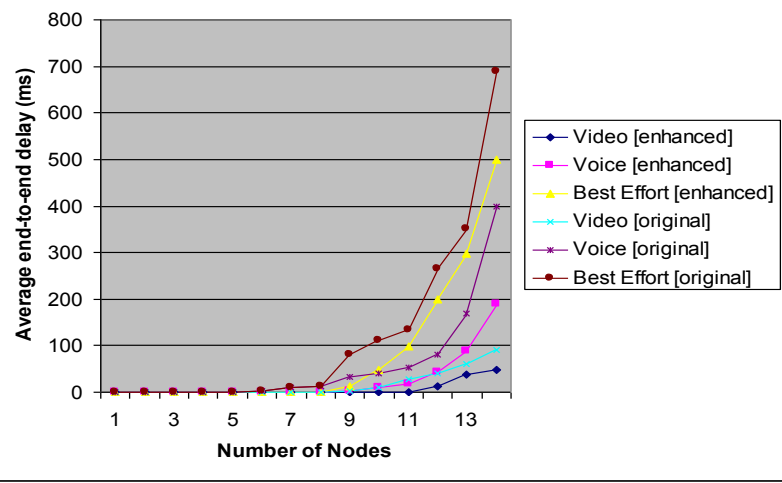

Figure 4 Effect of network load on the average end-to-end delay for different access categories (voice, video and best effort data) using original \& enhanced EDCA values.

Figures 4 illustrate the effect of increasing the number of active QoS stations transmitting data to the access point on the average end-to-end delay values for the three data flows separately from source (mobile stations) to destination (access point). It was modified the first scenario so that all the stations transmit three types of data flows. The channel load was varied by increasing the number of active QoS stations from 1 to 14. The enhanced CW size and AIFSN values illustrates better performance with respect to the video and voice flows, but not for the best effort data flow. This is shown in Figure 5 when the active QoS stations are 11.

As comparison, Figure 6 similarly represents the simulation results using the $\mathrm{CW}$ size and AIFSN values in Table 5. These enhanced values provide better results than ours with respect to best effort data flow. Here, the main concern is to enhance the performance for Video flow.

Another important factor that has a great effect on the IEEE802.11e WLAN performance for QoS support is the packet drop and loss ratio. To calculate the number of packets dropped or lost in the transmission medium, we subtract the number of packet successfully received by the receiver (the access point in our case) from the total number of packets sent by the sender (mobile stations).

In Figure 5, illustrates the effect of increasing the number of active QoS stations on the packet drop and loss ratio. The network load was varied by 3 stations at a time sending three different data flows. In this simulation, is to compare the original with the enhanced IEEE802.11e parameters.

It is clearly observed from Figure 6 the service differentiation between the different data flows according to their priority levels. This difference appears more when the channel is heavily loaded by increasing the number of stations. For the best effort data flow, the packet drop starts when the number of stations is 3 . That is due to the fact that best-effort data flow has the lowest priority. On the other hand, as the video flow is considered, the packet drop starts when the number of stations increases to 9 .

This reflects the fact that video flow has the highest priority to reserve the channel when it is heavily loaded. The percentage of the packet drop reaches up to $82 \%$ for the maximum channel load considering the best effort data flow, while it reaches up to $19 \%$ for the video flow.

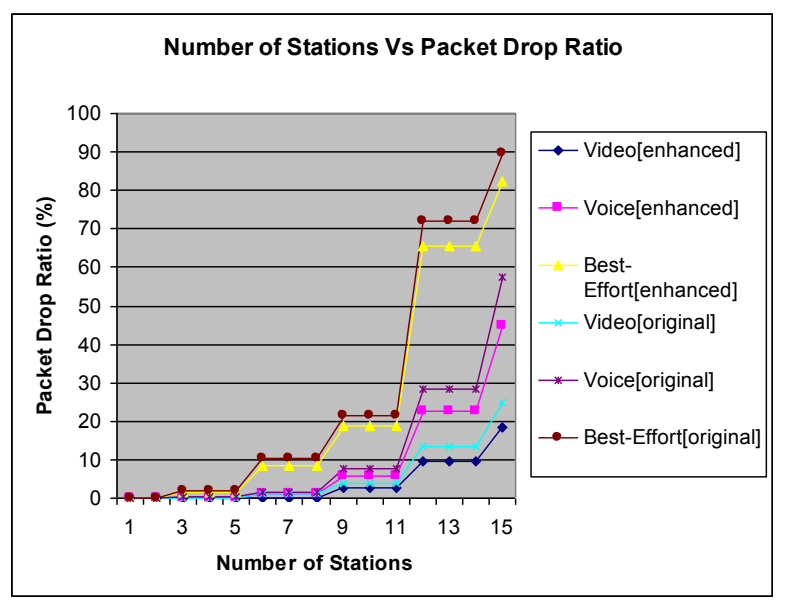

Figure 5 Effect of number of stations on the Packet drop ratio for different access categories (voice, video and best effort data) using original \& enhanced EDCA values.

In fact, the system throughput is inversely proportional to the number of stations. And the number of stations is inversely proportional to the dropped and lost packets. In addition, packet drop has great effect on the network average end-toend delay. Delay is directly proportional to the number of dropped packets.

\section{CONCLUSION}

Video transmission over wireless networks and the internet is a challenging task due to the stringent QoS required by video applications and also affected by many channel impairments. In this work, An Enhancement approaches of IEEE802.11e were presented. Different simulation scenarios such as average end-to-end delay, throughput and packet loss ratio to suite different environments under various conditions in performance analysis for MPEG-4 video transmission over WLANs were conducted. The level of performance such as 
Packet loss, PSNR, Decodable Frame Rate (Q) were evaluated which shows better results for lower packet loss, higher Throughput, PSNR \& Decodable Frame Rate (Q) for MPEG4 video transmission over IEEE802.11e. The experimentation results have shown that MPEG-4 video streaming services performs well only when the SNR is above $30 \mathrm{~dB}$. However, the received video quality becomes unacceptable in $20 \mathrm{~dB}$ to $30 \mathrm{~dB}$. Any traffic transmission will be easily denied when the SNR is below $20 \mathrm{~dB}$.

\section{REFERENCES}

[1] Vinod B Durdi, P. T. Kulkarni, and K. L. Sudha, Robust Video Transmission over Wireless Networks Using Cross Layer Approach" , Journal of Industrial and Intelligent Information Vol. 1, No. 2, June 2013:

[2] Kalvein Rantelobo, Wirawan, G. Hendrantoro, A. Affandi, and Hua-An Zhao, "A New Scheme for Evaluating Video Transmission over Broadband Wireless Network “, Future Wireless Networks and Information Systems, LNEE 143, pp. 335-341, Springerlink.com (C) Springer-Verlag Berlin Heidelberg 2012

[3] S.M. Koli, R.G. Purandare, S.P. Kshirsagar, and V.V. Gohokar, "A Survey on Video Transmission Using Wireless Technology “,CCSIT 2011, Part II, CCIS 132, pp. 137-147, Springer-Verlag Berlin Heidelberg 2011.

[4] Gopikrishnan.R , "An Efficient Real Time Video Multicasting Protocol and WLANs Cross-Layer Optimization in IEEE 802.11N “, IJCSMC, Vol. 3, Issue. 2, February 2014, pg.811 - 814

[5] S Kumar, Reactive and Proactive Routing Protocols for Wireless Mesh Network using Multimedia Streaming[A]. Proceedings of the International Conference on Recent Advances and Future Trends in Information Technology (iRAFIT 2012) (: International Journal of Computer Applications, Special Issue[C], 2012.

[6] T. Kim, "Scalable video streaming over internet", P h.D. Thesis, School of Electrical and Computer Engineering, Georgia Institute of Technology, Jan. 2005.

[7] D. Wu, Y. T. Hou, W. Zhu, Y.-Q. Zhang and J. M. Peha, "Streaming video over the internet: Approaches and directions," IEEE Trans. Circuits Syst. Video Technol., vol. 11, pp. 282-300, Mar. 2001.

[8] G. Conklin, G. Greenbaum, K. Lillevold, A. Lippman and Y. Reznik, "Video coding for streaming media delivery on the Internet," IEEE Trans. Circuits Syst. Video Technol., vol. 11, pp. 269-281, Mar. 2001.

[9] J.G. Apostolopoulos, W. Tan and S.J. Wee "Video Str eaming: Concepts, Algorithms, and Systems" Mobile and Media Systems L aboratory HP Laboratories Palo Alto, HPL-2002-260, Sept. 2002.

[10] F. Yang, Q. Zhang, W. Zhu and Y.Q. Zhang, "Bit Allo cation for Scalable Video Streaming over Mobile Wireless Internet", Infocom, 2004.

[11] F. Ziliani and J-C. Michelou, "Scalable Video Codin g in Digital Video Security", White paper, VisioWave, 2005.

[12] Y.-M. Hsiao, J.-F. Lee, J.-S. Chen, and Y.-S. Chu, "H.264 video transmissions over wireless networks: challenges and solutions," Computer Communications, vol. 34, no. 14, pp. 1661-1672, 2011. View at Publisher - View at Google Scholar · View at Scopus

[13] M. van der Schaar and N. S. Shankar, "Cross-layer wireless multimedia transmission: challenges, principles, and new paradigms," IEEE Wireless Communications, vol. 12, no. 4, pp. 50-58, 2005.

[14] Z. Han, G.-M. Su, A. Kwasinski, M. Wu, and K. J. R. Liu, "Multiuser distortion management of layered video over resource limited downlink multicode-CDMA," IEEE Transactions on Wireless Communications, vol. 5, no. 11, pp. 3056-3067, 2006. View at Publisher · View at Google Scholar · View at Scopus

[15] F. Fu and M. van der Schaar, "A systematic framework for dynamically optimizing multi-user wireless video transmission," IEEE Journal on Selected Areas in Communications, vol. 28, no. 3, pp. 308-320, 2010. View at Publisher - View at Google Scholar · View at Scopus

[16] F. Li, G. Liu, and L. He, "Cross-layer approach to multiuser H.264 video transmission over wireless networks," Journal of Multimedia, vol. 5, no. 2, pp. 110-117, 2010. View at Publisher - View at Google Scholar · View at Scopus

[17] D.-E. Meddour, A. Abdallah, T. Ahmed, and R. Boutaba, "A cross layer architecture for multicast and unicast video transmission in mobile broadband networks," Journal of Network and Computer Applications, vol. 35, no. 5, pp. 1377-1391, 2012. View at Publisher - View at Google Scholar · View at Scopus

[18] L. Superiori, M. Wrulich, P. Svoboda et al., "Contentaware scheduling for video streaming over HSDPA networks," in Proceedings of the 2nd International Workshop on Cross Layer Design (IWCLD '09), pp. 15, Palma de Mallorca, Spain, June 2009. View at Publisher · View at Google Scholar · View at Scopus

[19] A. Chan, H. Lundgren, and T. Salonidis, "Video-aware rate adaptation for MIMO WLANs," in Proceedings of the 19th IEEE International Conference on Network Protocols (ICNP '11), pp. 321-330, British Columbia, Canada, October 2011. View at Publisher - View at Google Scholar · View at Scopus

[20] J. Rexford, "Performance evaluation of smoothing algorithms for transmitting prerecorded variable-bit-rate video," IEEE Transactions on Multimedia, vol. 1, no. 3, pp. 302-312, 1999. View at Publisher · View at Google Scholar · View at Scopus

[21] A. Khalek, C. Caramanis, and R. Heath, "Video-aware MIMO precoding with packet prioritization and unequal modulation," in Proceedings of the 20th European Signal Processing Conference (EUSIPCO '12), pp. 1905-1909, Bucharest, Romania, August 2012.

[22] Ronak Dak, Dharm and Naveen Choudhary, A Technical Survey based on Secure Video Transmission Techniques, International Journal of Computer Applications.2014, Number 2, pp 19-23. 